\title{
ISOSPECTRAL DEFORMATIONS OF COMPACT SOLVMANIFOLDS
}

\author{
CAROLYN S. GORDON \& EDWARD N. WILSON
}

\section{Introduction}

Let $M$ and $M^{\prime}$ be compact Riemannian manifolds of the same dimension with Laplace-Beltrami operators $\Delta$ and $\Delta^{\prime} . M$ and $M^{\prime}$ are said to be isospectral if, for each $p \geqslant 0$, the collection of eigenvalues, with multiplicities, of $\Delta$ acting on smooth $p$-forms on $M$ coincides with the corresponding collection for $\Delta^{\prime}$. Milnor in 1961 gave a negative answer to the classical question "Are isospectral manifolds necessarily isometric?" by constructing two isospectral nonisometric flat 16-dimensional tori. Vignéras [12] and Ikeda [5] have constructed many examples of nonisometric manifolds for which the associated LaplaceBeltrami operators have the same spectra on functions, and Urakawa [11] has given counterexamples to the analogous problem for bounded domains in $\mathbf{R}^{n}$ when $n \geqslant 4$. All these examples involve finite families of manifolds. The goal of our paper is to construct continuous families of isospectral nonisometric manifolds. Our results contrast with an unpublished result of Kneser indicating that the number of isometry classes of flat tori isospectral to any given flat torus is finite and also contrasts with the proof of Guillemin and Kazhdan [3] that no compact surface of negative curvature admits a nontrivial isospectral deformation.

The manifolds we consider are of the form $(\Gamma \backslash G, g)$ where $G$ is an exponential solvable Lie group, $\Gamma$ is a uniform discrete subgroup, and $g$ is a Riemannian metric on $\Gamma \backslash G$ induced from a left invariant metric, also denoted by $g$, on $G$. As $\Phi$ ranges over a subgroup of $\operatorname{Aut}(G)$ which we call the group of "almost inner" automorphisms of $G$ and denote by $\operatorname{AIA}(G)$, we show that $(\Phi(\Gamma) \backslash G, g)$ is isospectral to $(\Gamma \backslash G, g)$. When $\Phi$ belongs to the group $\operatorname{Inn}(G)$ of inner automorphisms, it is easy to see that the two manifolds are isometric. However for $G$ nilpotent with $\operatorname{AIA}(G) \neq \operatorname{Inn}(G)$, we show that $\operatorname{AIA}(G)$ is topologically the product of $\operatorname{Inn}(G)$ and a Euclidean space $A$ and that the set $E$

Received September 9, 1983. 
of isometry classes of the manifolds $(\Phi(\Gamma) \backslash G, g), \Phi \in A$, may be naturally identified with the quotient of $A$ by an equivalence relation in which each equivalence class is discrete. Similar conclusions hold for certain solvable groups which are not nilpotent. Since $(\Phi(\Gamma) \backslash G, g)$ is isometric to $\left(\Gamma \backslash G, \Phi^{*} g\right)$ for $\Phi^{*} g$ the usual pullback of $g$ to another left invariant metric on $G$, our results may be also interpreted as providing a continuous family of isospectral nonisometric Riemannian structures on the fixed manifold $\Gamma \backslash G$.

The organization of the paper is as follows. $\$ 2$ defines the group $\operatorname{AIA}(G)$, proves a useful structure theorem for $\operatorname{AIA}(G)$ when $G$ is nilpotent, and studies a variety of examples. We establish the isospectrality of our manifolds for arbitrary exponential solvable $G$ on functions in $\$ 3$ and on $p$-forms $(p>0)$ in $\S 4$. $\$ 5$ begins with a necessary and sufficient condition for isometry of the manifolds in the case when all of the roots of the Lie algebra of $G$ are real and then restricts further to the case when $G$ is nilpotent to obtain the description of $E$ noted above. The section concludes by returning to the examples of $\S 2$. We look in particular at nilpotent groups of step 2 where we discover that in one case $C=\left\{\Phi \in A:(\Gamma \backslash G, g)\right.$ is isometric to $\left.\left(\Gamma \backslash G, \Phi^{*} g\right)\right\}$ reduces to a point while in another case $C$ is a lattice of full rank in $A$. Since $E$ in both cases consists of the orbits in $A / C$ under the action of a finite group, $E$ is essentially a Euclidean space in one case and a torus in the other. Our last example makes it clear that similar phenomena arise when $G$ is not nilpotent.

Readers interested in background information on the isospectral problem may wish to consult [1] or [10]. The authors are grateful to Richard Millman for suggesting that they try to apply their isometry group structure results to the isospectral problem on compact solvmanifolds.

\section{Almost inner automorphisms}

2.1. Notation. Let $G$ be a connected and simply connected Lie group with Lie algebra $g$. The centers of $G$ and $g$ will be denoted by $Z$ and $z$. Let $\operatorname{Aut}(G)$ (respectively, Aut $(\mathrm{g})$ ) be the group of all automorphisms of $G$ (respectively, $\mathfrak{g}$ ) and $\operatorname{Der}(g)$ the collection of all derivations of $g$. For $\Phi \in \operatorname{Aut}(G)$, the differential $\Phi_{*}: \mathfrak{g} \rightarrow \mathfrak{g}$ of $\Phi$ is in $\operatorname{Aut}(\mathfrak{g})$ and the assumptions above imply that $\Phi \rightarrow \Phi_{*}$ is an isomorphism from $\operatorname{Aut}(G)$ onto $\operatorname{Aut}(g)$. Aut $(g)$ is a Lie subgroup of the general linear group on $\mathrm{g}$.

Via the above map, we $\operatorname{regard} \operatorname{Aut}(G)$ as a Lie group whose Lie algebra is $\operatorname{Der}(\mathfrak{g})$. For $x \in G, I_{x}$ denotes the inner automorphism of $G$ defined by $I_{x}(y)=x y x^{-1}$ The collection $\operatorname{Inn}(G) \cong G / Z$ of all inner automorphisms of $G$ is a normal Lie subgroup of $\operatorname{Aut}(G)$ whose Lie algebra is ad $g \cong g / z$, where 
ad $X(Y)=[X, Y]$ for $X, Y \in g$. By definition, ad $g$ is the collection of all inner derivations of $g$.

We use exp as a generic symbol for the exponential map from Lie algebra to Lie group. In particular, exp: $\operatorname{Der}(g) \rightarrow \operatorname{Aut}(g)$ is the usual matrix exponential. Recall that for $x=\exp X \in G,\left(I_{x}\right)_{*}$ is denoted by $\operatorname{Ad}(\exp X)$ and coincides with $\exp (\operatorname{ad} X)$. We shall be concerned chiefly with exponential solvable groups, i.e., $G$ is solvable and exp: $g \rightarrow G$ is a diffeomorphism. We denote the inverse of exp by $\log : G \rightarrow \mathfrak{g}$. Recall that for $g$ solvable, $G$ is exponential solvable if and only if the imaginary part of each root of $g$ is a multiple of the real part of the root. In particular, every nilpotent $G$ is exponential solvable. For $G$ nilpotent, let $\left\{g^{j}: j \geqslant 1\right\}$ be the ideals in the lower central series for $\mathfrak{g}$. Thus $\mathfrak{g}=\mathfrak{g}^{1}, \mathfrak{g}^{j}=\left[\mathfrak{g}, \mathfrak{g}^{j-1}\right]$ for $j>1,\left[\mathfrak{g}^{j}, \mathfrak{g}^{k}\right] \subset \mathfrak{g}^{j+k}$, and $\mathrm{g}^{j}=\{0\}$ if and only if $j>m$ where $m$ is the step size of $\mathfrak{g}$. For $X, Y \in \mathfrak{g}$ and $Z=\log (\exp X \exp Y)$, the Campbell-Baker-Hausdorff formula

$$
Z=X+Y+\frac{1}{2}[X, Y]+\cdots
$$

expresses $Z$ as a polynomial of order $m$ in the variables $X$ and $Y$.

$\operatorname{Aut}(G)$ acts on both $\mathfrak{g}$ and $\mathfrak{g}^{*}$, the dual space of $\mathfrak{g}$, by $\Phi \cdot X=(\Phi)_{*}(X)$, $\Phi \cdot \lambda=\lambda \circ \Phi_{*}^{-1}$ for $\Phi \in \operatorname{Aut}(G), X \in \mathfrak{g}, \lambda \in \mathrm{g}^{*}$. By differentiation, $\operatorname{Der}(\mathrm{g})$ acts on these spaces as well. Denote by $\operatorname{Inn}(G) \cdot X, \operatorname{Inn}(G) \cdot \lambda, \operatorname{ad} \mathfrak{g} \cdot X=$ $[\mathrm{g}, X]$, and $\mathrm{ad} g \cdot \lambda=\lambda \circ$ ad $g$ the orbits of $\operatorname{Inn}(G)$ and ad $g$ under these actions.

We use Id as a generic symbol for the identity map on a space with the context of a discussion used to determine the space in question.

2.2. Definitions. (i) $\operatorname{AIA}(G)=\{\Phi \in \operatorname{Aut}(G): \Phi \cdot \lambda \in \operatorname{Inn}(G) \cdot \lambda$ for every $\left.\lambda \in \mathrm{g}^{*}\right\}$. The elements of $\mathrm{AIA}(G)$ are called almost inner automorphisms of $G$.

(ii) $\operatorname{AID}(\mathfrak{g})=\{\varphi \in \operatorname{Der}(\mathfrak{g}): \varphi(X) \in \operatorname{ad} \mathfrak{g} \cdot X$ for every $X \in \mathfrak{g}\}$. The elements of $\operatorname{AID}(g)$ are called almost inner derivations of $g$.

2.3. Theorem. Let $G$ be nilpotent of step $m$. Then

(i) $\operatorname{AIA}(G)$ is a closed normal Lie subgroup of $\operatorname{Aut}(G)$ with Lie algebra $\operatorname{AID}(g)$,

(ii) $\operatorname{AIA}(G)$ is a connected, simply connected, nilpotent group of step $m-1$,

(iii) $\operatorname{AIA}(G)=\{\Phi \in \operatorname{Aut}(G): \Phi \cdot X \in \operatorname{Inn}(G) \cdot X$ for every $X \in \mathfrak{g}\}$.

Proof. To avoid repetition in arguments, we temporarily use $V$ to denote either the vector space $g$ or $\mathfrak{g}^{*}$ and denote by $\tilde{G}=\tilde{G}(V)$ (respectively, $\tilde{\mathrm{g}}=\tilde{\mathrm{g}}(V))$ the maximal set in $\operatorname{Aut}(G)$ (respectively, $\operatorname{Der}(\mathfrak{g})$ ) whose orbits on $V$ coincide with those of $\operatorname{Inn}(G)$ (respectively, ad $\mathfrak{g}$ ). Thus for $V=\mathfrak{g}^{*}, \tilde{G}=$ $\operatorname{AIA}(G)$ and for $V=\mathfrak{g}, \tilde{\mathfrak{g}}=\operatorname{AID}(\mathfrak{g})$. Clearly $\tilde{G}$ is a normal subgroup of Aut $(G)$. Since $(\operatorname{ad} X)^{m}=0$ for every $X \in \mathfrak{g}, X \rightarrow \exp (\operatorname{ad} X) \cdot v$ is a polynomial map of order $m-1$ from the vector space $g$ to $V$ for every $v \in V$. The 
range of every such map is a closed set in $V$, i.e. all $\operatorname{Inn}(G)$ orbits in $V$ are closed. It follows that $\tilde{G}$ is closed in $\operatorname{Aut}(G)$ and thus a Lie subgroup. We will now show that $\exp (\tilde{g})=\tilde{G}$; given this, $\tilde{\mathfrak{g}}$ is the Lie algebra of $\tilde{G}$ and $\tilde{G}$ is connected. First note that the collection $T(G)$ consisting of all automorphisms $\Psi$ of $G$ such that $\Psi-$ Id maps $\mathrm{g}^{j}$ into $\mathrm{g}^{j+1}$ for all $j \geqslant 1$ is a nilpotent Lie subgroup of $\operatorname{Aut}(G)$ whose Lie algebra $\mathrm{t}(\mathfrak{g})$ consists of all derivations $\Psi$ mapping $\mathrm{g}^{j}$ into $\mathrm{g}^{j+1}$ for $j \geqslant 1$. Using the fact that the matrix exponential maps the lower triangular nilpotent matrices diffeomorphically onto the lower triangular unipotent matrices, it follows easily that $\exp (\mathrm{t}(\mathfrak{g}))=T(G)$. It is routine to check that for both choices of $V, \tilde{G} \subset T(G)$ and $\tilde{\mathfrak{g}} \subset \mathfrak{t}(\mathfrak{g})$. Now suppose $\Phi \in \tilde{G}$. Then $\Phi=\exp \varphi$ for some $\varphi \in \mathfrak{t}(\mathfrak{g})$. Given $v \in V$, there exists $X \in \mathrm{g}$ such that $v=(\exp (-\operatorname{ad} X) \exp \varphi) \cdot v$. By (1) in the group $T(G)$ and since $\operatorname{ad} g$ is an ideal in Der $\mathfrak{g}$ (indeed $[\varphi, \operatorname{ad} X]=\operatorname{ad} \varphi(X)$ ), we obtain $v=\exp (\varphi-\operatorname{ad} Y) \cdot v$ where $Y=X-\frac{1}{2} \varphi(X)+\cdots \in \mathrm{g}$. But then $(\varphi-\operatorname{ad} Y) \cdot v=0$ since we have $f(A) A \cdot v=0$ where $A=\varphi-\operatorname{ad} Y$ is a nilpotent operator and

$$
f(A)=\sum_{k=0}^{m-1} \frac{A^{k}}{(k+1) !}
$$

is invertible. Since $v$ was arbitrary, we conclude $\varphi \in \tilde{g}$ and $\exp \tilde{\mathfrak{g}} \supset \tilde{G}$. Conversely, suppose $\varphi \in \tilde{\mathrm{g}}, \Phi=\exp \varphi$, and $v \in V$. Selecting $Y$ for which $(\varphi-\operatorname{ad} Y) \cdot v=0$ and again using (1) in $T(G)$, we obtain $\Phi \cdot v=$ $\exp \varphi \exp (\operatorname{ad} Y-\varphi) \cdot v=\exp (\operatorname{ad} X) \cdot v$ for $X=Y+\frac{1}{2} \varphi(Y)+\cdots \in \mathrm{g}$. Thus $\Phi \in \tilde{G}$ and $\exp \tilde{g}=\tilde{G}$.

We now change notation and denote $\tilde{\mathfrak{g}}\left(\mathfrak{g}^{*}\right)$ by $\mathfrak{g}_{1}, \tilde{g}(\mathfrak{g})=\operatorname{AID}(\mathfrak{g})$ by $\mathfrak{g}_{2}$. Suppose $\varphi \in \mathfrak{g}_{1}$ and $X \in \mathfrak{g}$; if $\varphi(X) \notin[\mathfrak{g}, X]$, there exists $\lambda \in \mathfrak{g}^{*}$ vanishing on $[\mathfrak{g}, X]$ but with $\lambda(\varphi(X)) \neq 0$. Since $\lambda \circ \varphi=(-\varphi) \cdot \lambda=(-\operatorname{ad} Y) \cdot \lambda=$ $\lambda \circ$ ad $Y$, this is a contradiction. Thus $\mathfrak{g}_{1} \subset \mathfrak{g}_{2}$. By duality of $\mathfrak{g}$ and $\mathfrak{g}^{*}$, we similarly obtain $g_{2} \subset g_{1}$ and conclude $g_{1}=g_{2}$. This concludes the proof of the remaining part of (i) and also proves (iii) since our argument above yields

$$
\operatorname{AIA}(G)=\exp g_{1}=\exp g_{2}=\text { right-hand side of (iii). }
$$

For (ii), all that remains to be noted is that $\operatorname{Inn}(G) \subset \operatorname{AIA}(G) \subset T(G)$ and both $\operatorname{Inn}(G)$ and $T(G)$ are of step $m-1$.

2.4. Examples. In the following examples, $\mathfrak{g}$ is defined by giving a basis $\mathscr{B}$ and bracket relations among the basis elements with rational structure constants. In anticipation of $\S 5$, we note that in Example (i), $\operatorname{AID}(g)$ has a basis $\mathcal{T}$ whose matrices relative to $\mathscr{B}$ are rational while in Example (ii), the matrix relative to $\mathscr{B}$ of every non-inner element of $\operatorname{AID}(g)$ has at least one irrational entry. 
(i) Let $\mathscr{B}=\left\{X_{i}, Y_{i}, Z_{i}: 1 \leqslant i \leqslant 2\right\}$ with $\left[X_{1}, Y_{1}\right]=Z_{1}=\left[X_{2}, Y_{2}\right],\left[X_{1}, Y_{2}\right]=$ $Z_{2}$, and all other brackets zero. Then $z=\mathbf{R} Z_{1}+\mathbf{R} Z_{2}$ has dimension 2 so $\operatorname{dim}(\operatorname{adg})=\operatorname{dim} g / z=4$. If $U \in \mathfrak{g}$ with $U \notin z$, it is easy to check that $[\mathfrak{g}, U]=\mathbf{R} Z_{1}$ if $U \in \mathbf{R} X_{2}+\mathbf{R} Y_{1}+\mathfrak{z}$ and otherwise $[\mathfrak{g}, U]=[\mathfrak{g}, \mathfrak{g}]=\mathfrak{z}$. It follows that

$$
\operatorname{AID}(\mathrm{g})=\left\{\varphi \in \operatorname{Hom}(\mathrm{g}, \mathrm{z}):\left.\varphi\right|_{\mathrm{z}}=0 \text { and } \varphi\left(X_{2}\right), \varphi\left(Y_{1}\right) \in \mathbf{R} Z_{1}\right\} .
$$

Define $\varphi_{1}, \varphi_{2} \in \operatorname{AID}(g)$ by insisting that $\varphi_{1}\left(X_{1}\right)=Z_{2}=\varphi_{2}\left(Y_{2}\right)$ with $\varphi_{1}$ and $\varphi_{2}$ zero on the remaining elements of $\mathscr{\Re}$. By a trivial computation, $\mathscr{T}=\left\{\operatorname{ad} X_{i}\right.$, $\left.\operatorname{ad} Y_{i}, \varphi_{i}: i=1,2\right\}$ is a basis of $\operatorname{AID}(\mathfrak{g})$. Since $m$ is $2, \operatorname{AIA}(G)$ is abelian and may be viewed as the direct product of $\operatorname{Inn}(G) \cong \mathbf{R}^{4}$ with $\left\{\operatorname{Id}+t_{1} \varphi_{1}+t_{2} \varphi_{2}\right.$ : $\left.t_{1}, t_{2} \in \mathbf{R}\right\} \cong \mathbf{R}^{2}$.

(ii) Let $\mathscr{B}=\left\{X_{i}, Y_{j}, Z_{k}: 1 \leqslant i \leqslant 2,1 \leqslant j, k \leqslant 3\right\}$ with $\left[X_{1}, Y_{j}\right]=Z_{j}$ for $j=1,2,3,\left[X_{2}, Y_{1}\right]=Z_{3},\left[X_{2}, Y_{2}\right]=2 Z_{1},\left[X_{2}, Y_{3}\right]=Z_{2}$, and all other brackets zero. Thus $\mathrm{g}$ is again two-step nilpotent with $[\mathrm{g}, \mathrm{g}]=\mathrm{z}=\mathbf{R} Z_{1}+\mathbf{R} Z_{2}+\mathbf{R} Z_{3}$ three dimensional so $\operatorname{dim}(\operatorname{adg})=5$. Set $\mathfrak{g}_{1}=\mathbf{R} X_{1}+\mathbf{R} X_{2}$ and $\mathfrak{g}_{2}=\mathbf{R} Y_{1}+$ $\mathbf{R} Y_{2}+\mathbf{R} Y_{3}$. Note that $g_{1}$ and $g_{2}$ are abelian with $[g, g]=\left[g_{1}, g_{2}\right]$. In order for a linear map $\varphi: \mathfrak{g} \rightarrow \mathfrak{g}$ to be in $\operatorname{AID}(\mathfrak{g})$, it is necessary and sufficient that

(a) $\varphi($ g) $\subset$ z,$\varphi(\mathfrak{z})=\{0\}$;

(b) for each $Y \in \mathrm{g}_{2}$, there exists $X \in \mathrm{g}_{1}$ such that $\varphi(Y)=[X, Y]$;

(c) for each $X \in \mathfrak{g}_{1}$, there exists $Y \in \mathrm{g}_{2}$ such that $\varphi(Y)=[X, Y]$.

An easy calculation shows that $\varphi$ can satisfy (b) only if $X$ may be chosen independently of $Y$, i.e., $\left.\varphi\right|_{g_{2}}=$ ad $\left.X\right|_{g_{2}}$ for some $X \in \mathfrak{g}_{1}$. However, (c) allows richer possibilities. Let $\left.\varphi\right|_{g_{1}}$ be defined by

$$
\begin{aligned}
& \varphi\left(X_{1}\right)=\alpha_{1} Z_{1}+\alpha_{2} Z_{2}+\alpha_{3} Z_{3}=\left[X_{1}, \alpha_{1} Y_{1}+\alpha_{2} Y_{2}+\alpha_{3} Y_{3}\right], \\
& \varphi\left(X_{2}\right)=2 \beta_{2} Z_{1}+\beta_{3} Z_{2}+\beta_{1} Z_{3}=\left[X_{2}, \beta_{1} Y_{1}+\beta_{2} Y_{2}+\beta_{3} Y_{3}\right] .
\end{aligned}
$$

For $X=x_{1} X_{1}+x_{2} X_{2}$ with $x_{1}^{3}+2 x_{2}^{3} \neq 0$, it is easy to check that $\left[X, g_{2}\right]=z$ and thus (c) can be satisfied; however if $x_{1}^{3}+2 x_{2}^{3}=0$ with $x_{1} \neq 0$, (c) is satisfied if and only if

$$
c^{2}\left(\alpha_{1}-\beta_{1}\right)+c\left(\alpha_{3}-\beta_{3}\right)+\left(\alpha_{2}-\beta_{2}\right)=0,
$$

where $c=2^{-1 / 3}$. It follows that $\operatorname{dim} \operatorname{AID}(g)=7$ with a typical element $\varphi$ described uniquely by an element $X \in \mathrm{g}_{1}$ and a solution of (3); $\varphi$ is inner if and only if $\alpha_{i}=\beta_{i}$ for $i=1,2,3$. Since $1, c$, and $c^{2}$ are independent over the rationals, $\varphi$ has rational matrix entries relative to $\mathscr{B}$ if and only if $\varphi$ is inner.

(iii) Let $n \geqslant 3$ and $\Re=\left\{X_{1}, X_{2}, Y_{i}: 1 \leqslant i \leqslant n\right\}$ where $\left[X_{1}, Y_{i}\right]=Y_{i+1}$ for $1 \leqslant i \leqslant n-1,\left[X_{1}, Y_{n}\right]=0=\left[X_{1}, X_{2}\right]$, and ad $X_{2}$ is a polynomial in ad $X_{1}$ without constant term and with degree between 2 and $n-1$. Here $g$ is nilpotent of step $n$. It is easy to check that $\operatorname{AID}(\mathfrak{g})$ is the direct sum of ad $\mathfrak{g}$ 
and $\mathbf{R} \varphi$ where $\varphi\left(X_{2}\right)=Y_{n}$ and $\varphi$ is zero on the remaining elements of $\mathscr{B}$. It is trivial to construct more general examples of this kind where $\operatorname{dim}(\operatorname{AID}(\mathfrak{g}) / \mathbf{a d} \mathfrak{g})$ is arbitrarily large. One again takes $\mathfrak{g}$ as the semidirect product of an abelian algebra $a$ and an abelian ideal $\mathfrak{b}$ with ad $\mathfrak{a}$ acting by commuting nilpotent operators on $\mathfrak{b}$ but without the stipulation that the action be cyclic.

(iv) Let $\mathfrak{n}$ be the Lie algebra in Example (i) and $\mathfrak{g}$ the vector space direct sum of $\mathfrak{m}=\operatorname{span}\left\{H, X_{3}, Y_{3}\right\}$ and $\mathfrak{n}$ with $[\mathfrak{m}, \mathfrak{n}]=\{0\},\left[H, X_{3}\right]=X_{3},\left[H, Y_{3}\right]=$ $-Y_{3}$, and $\left[X_{3}, Y_{3}\right]=Z_{1}$. Here $g$ is solvable with roots \pm 1 and thus $G$ is exponential solvable. Extend the non-inner elements $\varphi_{1}, \varphi_{2} \in \operatorname{AID}(\mathfrak{n})$ to maps $\psi_{1}, \psi_{2}$ on $g$ by insisting that $\left.\psi_{i}\right|_{m}=\{0\}$ for $i=1,2$. It is trivial to check that $\psi_{1}, \psi_{2}$ belong to $\operatorname{AID}(\mathfrak{g})$ and that $\operatorname{AID}(\mathfrak{g})$ is the direct sum of ad $g$ and $\mathbf{R} \psi_{1}+\mathbf{R} \psi_{2}$.

(v) For the $(2 n+1)$-dimensional Heisenberg algebra $\mathfrak{g}=\mathfrak{h}_{n}$ (defined by $\mathscr{B}=\left\{X_{i}, Y_{i}, Z: 1 \leqslant i \leqslant n\right\}$ with $\left[X_{i}, Y_{i}\right]=Z$ and all other brackets zero), it is easy to check that $\operatorname{AID}(\mathfrak{g})=\operatorname{ad} \mathfrak{g}$. By a tedious calculation, $\operatorname{AID}(\mathfrak{g})=\mathrm{ad} \mathfrak{g}$ as well for $g$ the Lie algebra of all lower triangular $n \times n$ matrices.

\section{Isospectral metrics}

3.1. Notation. Let $G=\exp g$ be exponential solvable and assume that $G$ admits a uniform discrete subgroup $\Gamma$, i.e. a discrete subgroup $\Gamma$ such that $\Gamma \backslash G$ is compact. For $G$ nilpotent, this is equivalent to the existence of a basis for $\mathfrak{g}$ relative to which the structure constants are rational (see $[8, \mathrm{p}$. 34]). Thus Examples 2.4(i)-(iii) apply, provided that the polynomial in (iii) has rational coefficients. We shall see in $\S 5$ that Example 2.4(iv) applies as well.

Each left-invariant Riemannian metric $g$ on $G$ induces uniquely a Riemannian metric, again denoted by $g$, on $\Gamma \backslash G$ such that the natural projection map $\pi:(G, g) \rightarrow(\Gamma \backslash G, g)$ is a Riemannian covering. The elements of $G$ then define local isometries of $(\Gamma \backslash G, g)$ via the locally defined left translation action of $G$ on $\Gamma \backslash G$. Thus $(\Gamma \backslash G, g$ ) is a locally homogeneous, compact, Riemannian solvmanifold. Note that while $\Gamma \backslash G$ is homogeneous under the right translation action $R_{\Gamma, y}(\Gamma x)=\Gamma x y(x, y \in G), R_{\Gamma, y}$ is not in general an isometry on $(\Gamma \backslash G, g)$ since $g$ was chosen to be left invariant. Let $\Omega_{g}$ be the Riemannian volume element on $G$ or $\Gamma \backslash G$ and $L^{2}(\Gamma \backslash G)$ the space of measurable functions on $\Gamma \backslash G$ which are square integrable with respect to $\Omega_{g}$. On $G$, left invariance of $g$ implies that $\Omega_{g}$ is a left Haar measure. However, the existence of $\Gamma$ forces $G$ to be unimodular so $\Omega_{g}$ is also a right Haar measure on $G$. On $\Gamma \backslash G, \Omega_{g}$ is invariant under the right translations $R_{\Gamma, y}$. Let $\rho_{\Gamma}(y) f=$ $f \circ R_{\Gamma, y}$ for $f \in L^{2}(\Gamma \backslash G), y \in G$ and $\left(\rho_{\Gamma}\right)_{*}(Y) f=(d / d t)_{t=0}\left(\rho_{\Gamma}(\exp t Y) f\right)$ 
for $Y \in \mathfrak{g}$ and $f \in C^{\infty}(\Gamma \backslash G)$. Thus $y \rightarrow \rho_{\Gamma}(y)$ is a unitary representation of $G$ on $L^{2}(\Gamma \backslash G)$ and $Y \rightarrow\left(\rho_{\Gamma}\right)_{*}(Y)$ is a skew-adjoint representation of $g$ on the dense subspace $C^{\infty}(\Gamma \backslash G)$ of $L^{2}(\Gamma \backslash G)$. Since Haar measure on $G$ is unique up to scalar multiple, $L^{2}(\Gamma \backslash G)$ does not depend on the choice of $g$.

Let $\Delta_{g}$ be the Laplace-Beltrami operator on $\Gamma \backslash G$. As will be reviewed in 4.1, $\Delta_{g}$ is an operator on the space of smooth exterior forms on $\Gamma \backslash G$ which preserves the degree of a form and is self-adjoint with respect to an inner product on forms arising naturally from $g$. In this section, it will be enough to know that $\Delta_{g}$ acts on $C^{\infty}(\Gamma \backslash G)$ by

$$
\Delta_{g}=-\sum_{i=1}^{n}\left[\left(\rho_{\Gamma}\right)_{*}\left(Y_{i}\right)\right]^{2}
$$

for $Y_{1}, Y_{2}, \cdots, Y_{n}$ any basis of $\mathfrak{g}$ which is orthonormal with respect to the inner product on $g$ induced by $g$. Indeed, (1) follows by an easy computation using only formula 4.2(5) and the interpretation of each $Y \in g$ as a smooth vector field on $\Gamma \backslash G$ acting on $C^{\infty}(\Gamma \backslash G)$ by $\left(\rho_{\Gamma}\right)_{*}(Y)$.

For any $\Phi \in \operatorname{Aut}(G), \Phi^{*} g$ is another left invariant metric on $g$ defined by $\left(\Phi^{*} g\right)(X, Y)=g\left(\Phi_{*} X, \Phi_{*} Y\right)$ for $X, Y \in g$.

3.2. Lemma. With the notations of 3.1, for $\Phi \in \operatorname{AIA}(G)$, the representations $\rho_{\Gamma}$ and $\rho_{\Gamma} \circ \Phi$ are unitarily equivalent, i.e. there exists a unitary operator $T$ on $L^{2}(\Gamma \backslash G)$ such that

$$
T \rho_{\Gamma}(y)=\rho_{\Gamma}(\Phi(y)) T \text { for all } y \in G
$$

Proof. We digress for a moment to review the Kirillov or orbit theory of unitary representations of $G$. Details for the case $G$ nilpotent may be found in [7]; see [6] for the exponential solvable case. To each $\lambda \in g^{*}$ there is associated an irreducible unitary representation $\pi_{\lambda}$ of $G$ with the following properties:

(i) For $\lambda, \mu \in g^{*}, \pi_{\lambda} \sim \pi_{\mu} \Leftrightarrow \mu \in \operatorname{Inn}(G) \cdot \lambda$ where $\sim$ denotes unitary equivalence and $\operatorname{Inn}(G) \cdot \lambda$ is defined as in $\$ 2.1$.

(ii) For every irreducible unitary representation $\sigma$ on $G$, there exists $\lambda \in g^{*}$ such that $\sigma \sim \pi_{\lambda}$.

(iii) $\pi_{\lambda} \circ \Psi=\pi_{\lambda} \circ \Psi_{*}$ whenever $\lambda \in g^{*}, \Psi \in \operatorname{Aut}(G)$.

Let $Q$ be a complete collection of orbit representations for $\operatorname{Inn}(G)$ in $\mathfrak{g}^{*}$. Since $\Gamma \backslash G$ is compact, $\rho_{\Gamma}$ decomposes discretely, i.e. $\rho_{\Gamma} \sim \bigoplus_{\lambda \in \mathbb{Q}} m_{\lambda} \pi_{\lambda}$ where the multiplicity function $\lambda \rightarrow m_{\lambda}$ has values in the set of nonnegative integers and is zero off a countable subset of $\mathcal{Q}$. By (iii) $\rho_{\Gamma} \circ \Phi \sim \bigoplus_{\lambda \in \mathcal{Q}} m_{\lambda} \pi_{\lambda} \circ \Phi_{*}$. However, by Definition 2.2, $\lambda \circ \Phi_{*} \in \operatorname{Inn}(G) \cdot \lambda$ for every $\lambda$ and the lemma follows.

3.3. Definition. For $M$ a compact Riemannian manifold and $0 \leqslant p \leqslant$ $\operatorname{dim} M$, let $\operatorname{spec}_{p}(M)$ be the collection of eigenvalues, with multiplicities, of the 
Laplace-Beltrami operator $\Delta$ on $M$ acting on the space of smooth $p$-forms on $M$. Manifolds $M$ and $M^{\prime}$ are said to be isospectral if $\operatorname{spec}_{p}(M)=\operatorname{spec}_{p}\left(M^{\prime}\right)$ for all $p$.

3.4. Theorem. With the notations of 3.1, $\left(\Gamma \backslash G, \Phi^{*} g\right)$ and $(\Gamma \backslash G, g)$ are isospectral for $\Phi \in \operatorname{AIA}(G)$.

Proof. The proof for $p=0$ will be given here with the proof for $p \geqslant 0$ postponed to $\S 4$. To simplify notation, replace $\Phi$ by $\Phi^{-1}$ in the statement of the theorem and write $g^{\prime}$ for $\left(\Phi^{-1}\right)^{*} g, \Delta$ for $\Delta_{g}, \Delta^{\prime}$ for $\Delta_{g^{\prime}}$.

Let $Y_{1}, Y_{2}, \cdots, Y_{n}$ be a basis for $g$ which is orthonormal relative to $g$. Then $\Delta$ is given by (1). Since $\Phi_{*} Y_{1}, \cdots, \Phi_{*} Y_{n}$ is a basis of $g$ orthonormal relative to $g^{\prime}$,

$$
\Delta^{\prime}=-\sum_{i=1}^{n}\left[\left(\rho_{\Gamma} \circ \Phi\right)_{*}\left(Y_{i}\right)\right]^{2}
$$

For $T$ as in 3.3(2) we have $T \circ\left(\rho_{\Gamma}\right)_{*}=\left(\rho_{\Gamma} \circ \Phi\right)_{*} Y_{i} \circ T$. By (1) and (3), $T \circ \Delta=\Delta^{\prime} \circ T$ so on $C^{\infty}(\Gamma \backslash G), \Delta^{\prime}=T \circ \Delta \circ T^{-1}$ is similar to $\Delta$ and therefore has the same eigenvalues.

3.5. Remarks. For $\Phi=I_{x}$ an inner automorphism of $G,(\Gamma \backslash G, g)$ and $\left(\Gamma \backslash G, \Phi^{*} g\right)$ are not only isospectral but isometric. Indeed, $I_{x}=L_{x} \circ R_{x^{-1}}$ for $L_{x}$ and $R_{x^{-1}}$ the left and right translates by $x$ on $G$. Since $g$ is left invariant, $\Phi^{*} g=R_{x^{-1}}^{*} g$ and $R_{\Gamma, x}:(\Gamma \backslash G, g) \rightarrow\left(\Gamma \backslash G, \Phi^{*} g\right)$ is an isometry. We shall see in $\S 5$ that if $\Phi$ is almost inner but not inner, then $(\Gamma \backslash G, g)$ and $\left(\Gamma \backslash G, \Phi^{*} g\right)$ are rarely isometric. Thus as $\Phi$ ranges over appropriate subsets in $\operatorname{AIA}(G)$ $\operatorname{Inn}(G)$, we will obtain a continuous deformation of $g$ by metrics $\Phi^{*} g$ with $(\Gamma \backslash G, g)$ and $\left(\Gamma \backslash G, \Phi^{*} g\right)$ isospectral but nonisometric. Alternatively, any $\Psi \in \operatorname{Aut}(G)$ induces an isometry from $\left(\Gamma \backslash G, \Psi^{*} g\right)$ to $(\Psi(\Gamma) \backslash G, g)$ so we obtain similar conclusions by fixing $g$ and continuously deforming $\Gamma$ within the class of uniform discrete subgroups of $G$. The latter approach is analogous to Milnor's famous example [1] of lattices $\Gamma_{1}$ and $\Gamma_{2}$ in $\mathbf{R}^{16}$ such that the tori $\Gamma_{1} \backslash \mathbf{R}^{16}$ and $\Gamma_{2} \backslash \mathbf{R}^{16}$ are isospectral but nonisometric when both are equipped with the standard flat metric; no continuous isospectral deformation of $\Gamma_{1}$ to $\Gamma_{2}$ is possible in this example, however.

\section{The Laplacian on $p$-forms}

4.1. Remarks. The goal of this section is to complete the proof of Theorem 3.4 , i.e. show that $\operatorname{spec}_{p}(\Gamma \backslash G, g)=\operatorname{spec}_{p}\left(\Gamma \backslash G, g^{\prime}\right)$ for $p>0$. As in the $p=0$ case, we shall produce an operator $T_{p}$ acting on smooth $p$-forms on $\Gamma \backslash G$ such that $T_{p} \circ \Delta=\Delta^{\prime} \circ T_{p}$. Prior to this, we need a workable formula for the action of $\Delta$ on $p$-forms. The formula needed applies to any compact oriented 
Riemannian manifold $M$ and is presumably well known to experts. Unfortunately we do not know a reference for this formula and will therefore prove it in \$4.3.

4.2. Notation and review. Let $M$ be an $n$-dimensional compact oriented Riemannian manifold with $\mathfrak{X}(M)$ the smooth vector fields on $M$ and $E(M)=$ $\sum_{p=0}^{n} E^{p}(M)$ the exterior algebra of smooth differential forms on $M$. For $X \in \mathfrak{X}(M)$ the Lie derivative operator $L_{X}$ and the covariant derivative operator $\nabla_{X}$ are derivations of the full tensor algebra on $M$ which commute with contractions, act by $X$ on functions, and are defined on vector fields (see [4]) by

$$
\begin{gathered}
L_{X}(Y)=[X, Y], \\
2\left\langle\nabla_{X} Y, Z\right\rangle=\quad X\langle Y, Z\rangle+\langle[X, Y], Z\rangle-\langle Y,[X, Z]\rangle \\
+Y\langle X, Z\rangle-Z\langle X, Y\rangle-\langle X,[Y, Z]\rangle,
\end{gathered}
$$

where $\langle\cdot, \cdot\rangle: \mathfrak{X}(M) \times \mathfrak{X}(M) \rightarrow C^{\infty}(M)$ expresses the Riemannian metric on $M$. On $E(M)$ are defined the exterior differential operator $d$ and, for $X \in$ $\mathfrak{X}(M)$, the interior multiplication operator $i(X)$. The operator $i(X)$ is a derivation of $E(M)$ of degree -1 which is defined on 1-forms by $i(X) \alpha=\alpha(X)$. On $E(M)$, one has

$$
L_{X}=\operatorname{di}(X)+i(X) d .
$$

The metric defines an isomorphism \#: $E^{1}(M) \rightarrow \mathfrak{X}(M)$ by $\langle \# \lambda, X\rangle=\lambda(X)$ for $\lambda \in E^{1}(M), X \in \mathfrak{X}(M)$. By definition, $\#(d f)=\operatorname{grad} f$ for $f \in E^{0}(M)=$ $C^{\infty}(M)$. It is easy to check that $\#$ commutes with $\nabla_{X}$ for every $X \in \mathfrak{X}(M)$. The metric on $\mathfrak{X}(M)$ is translated to one on $E(M)$ by insisting that $\langle \# \alpha, \# \beta\rangle=\langle\alpha, \beta\rangle$ for $\alpha, \beta \in E^{1}(M)$, that $E^{p}(M)$ be orthogonal to $E^{q}(M)$ for $p \neq q$, and that

$$
\left\langle\alpha_{1} \wedge \cdots \wedge \alpha_{p}, \beta_{1} \wedge \cdots \wedge \beta_{p}\right\rangle=\operatorname{det}\left(\left\langle\alpha_{i}, \beta_{j}\right\rangle\right)_{1 \leqslant i, j \leqslant p}
$$

for $\alpha_{1}, \cdots, \beta_{p} \in E^{1}(M)$. For $\Omega \in E^{n}(M)$ the Riemannian volume element on $M,(\alpha \mid \beta)=\int_{M}\langle\alpha, \beta\rangle \Omega$ defines an inner product on $E(M)$. The adjoint of $d$ relative to $(\cdot \mid \cdot)$ is denoted by $\delta$ and the Laplace-Beltrami operator $\Delta$ on $M$ is defined by

$$
\Delta=d \delta+\delta d .
$$

Using (4), it is easy to check that for $\alpha \in E^{1}(M)$, the adjoint of $i(\# \alpha)$ relative to $(\cdot \mid \cdot)$ is the exterior multiplication operator $\epsilon(\alpha)$ defined by $\epsilon(\alpha) \beta=\alpha \wedge \beta$. By (3), the adjoint $L_{X}^{*}$ of $L_{X}$ is therefore given by

$$
L_{X}^{*}=\delta \epsilon\left(\#^{-1} X\right)+\epsilon\left(\#^{-1} X\right) \delta .
$$


4.3. Proposition. With the notation of $\$ 4.2$,

(7) $\Delta(f \lambda)=(\Delta f) \lambda+f(\Delta \lambda)-2 \nabla_{\operatorname{grad} f} \lambda$ for $f \in C^{\infty}(M), \lambda \in E(M)$.

Proof. By a routine calculation using only the properties noted in $\$ 4.2$ (see [9])

$$
\delta(f \lambda)=-i(\operatorname{grad} f) \lambda+f(\delta \lambda) .
$$

Using (3), (5), (6), and (8), it follows directly that

$$
\Delta(f \lambda)=f(\Delta \lambda)-\left(L_{\operatorname{grad} f}-L_{\operatorname{grad} f}^{*}\right) \lambda .
$$

From (1), for $\alpha, \beta \in E^{1}(M)$, we obtain, with the aid of $\langle\alpha, \beta\rangle=\alpha(\# \beta)=$ $\beta(\# \alpha)$,

$$
\left\langle L_{\operatorname{grad} f} \alpha, \beta\right\rangle-\left\langle\alpha, L_{\operatorname{grad} f} \beta\right\rangle=\langle \# \beta,[\operatorname{grad} f, \# \alpha]\rangle-\langle \# \alpha,[\operatorname{grad} f, \# \beta]\rangle .
$$

Since $\langle\operatorname{grad} f, U\rangle=U f$ for $U \in \mathfrak{X}(M)$, the last 3 terms in (2) collapse to zero when $X=\operatorname{grad} f$. Using this and $\left\langle\nabla_{\operatorname{grad} f} \alpha, \beta\right\rangle=\left\langle\nabla_{\operatorname{grad} f} \# \alpha, \# \beta\right\rangle$, it follows that

$$
\begin{aligned}
\left\langle L_{\operatorname{grad} f} \alpha, \beta\right\rangle-\left\langle\alpha, L_{\operatorname{grad} f} \beta\right\rangle-2\left\langle\nabla_{\operatorname{grad} f} \alpha, \beta\right\rangle & \\
& =-\operatorname{grad} f\langle\alpha, \beta\rangle=-\langle d f, d\langle\alpha, \beta\rangle\rangle .
\end{aligned}
$$

Now let $\lambda=\alpha_{1} \wedge \cdots \wedge \alpha_{p}, \mu=\beta_{1} \wedge \cdots \wedge \beta_{p}$ be elementary $p$-forms on $M$ and define $m_{i j}=\left\langle\alpha_{1} \wedge \cdots \wedge \hat{\alpha}_{i} \wedge \cdots \wedge \alpha_{p}, \beta_{1} \wedge \cdots \wedge \hat{\beta}_{j} \wedge \cdots \beta_{p}\right\rangle$ where ${ }^{\wedge}$ denotes a deleted term. Since $L_{\text {grad } f}$ and $\nabla_{\text {grad } f}$ are derivations of degree 0 on $E(M)$, (4), (10), and the classical formula for differentiating a determinant yield

$$
\begin{aligned}
& \left(\left(L_{\operatorname{grad} f}-L_{\operatorname{grad} f}^{*}-2 \nabla_{\operatorname{grad} f}\right) \lambda \mid \mu\right) \\
& \quad=\sum_{i, j=1}^{p}(-1)^{i+j} \int_{M}\left\{\left\langle\left(L_{\operatorname{grad} f}-2 \nabla_{\operatorname{grad} f}\right) \alpha_{i}, \beta_{j}\right\rangle-\left\langle\alpha_{i}, L_{\operatorname{grad} f} \beta_{j}\right\rangle\right\} m_{i j} \Omega \\
& \quad=-(d f \mid d\langle\lambda, \mu\rangle)=(-\delta d f \mid\langle\lambda, \mu\rangle)=-((\Delta f) \lambda \mid \mu) .
\end{aligned}
$$

Thus we obtain

$$
\left(L_{\operatorname{grad} f}-L_{\operatorname{grad} f}^{*}\right) \lambda=2 \nabla_{\operatorname{grad} f} \lambda-(\Delta f) \lambda
$$

for $\lambda$ an elementary $p$-form in $E(M)$. But then (11) holds for every $\lambda \in E(M)$ and (7) is immediate from (9) and (11).

4.4. Proof of Theorem 3.4, continued. We use the same notations as in $\$ 3.4$. As noted in $\$ 3.1$, elements in $g$ may be viewed as elements of $\mathfrak{X}(\Gamma \backslash G)$. Similarly elements of $\Lambda^{P}\left(\mathrm{~g}^{*}\right)$ may be viewed first as left invariant $p$-forms on $G$ 
and then as elements of $E^{p}(\Gamma \backslash G)$. With this interpretation, $E^{p}(\Gamma \backslash G)=$ $C^{\infty}(\Gamma \backslash G) \otimes \Lambda^{p}\left(g^{*}\right)$ and we may define $T_{p}: E^{p}(\Gamma \backslash G) \rightarrow E^{p}(\Gamma \backslash G)$ by $T_{p}=T$ $\otimes\left(\Phi^{-1}\right)^{*}$ where $T$ is the operator found in Lemma 3.2. In other words, for $\lambda_{1}, \cdots, \lambda_{r}$ any basis of $\Lambda^{p}\left(\mathrm{~g}^{*}\right)$, every $\lambda \in E^{p}(\Gamma \backslash G)$ is expressible in the form $\lambda=\sum_{i=1}^{r} f_{i} \lambda_{i}$ for $f_{1}, \cdots, f_{r} \in C^{\infty}(\Gamma \backslash G)$ and

$$
T_{p} \lambda=\sum_{i=1}^{r}\left(T f_{i}\right)\left(\Phi^{-1}\right)^{*} \lambda_{i}
$$

Trivially the spaces $\Lambda^{p}\left(\mathrm{~g}^{*}\right) \subset E^{p}(\Gamma \backslash G)$ are invariant under the operators $\Delta, \Delta^{\prime}$ and under $\nabla_{X}, \nabla_{X}^{\prime}$ for $X \in \mathfrak{g}$, where $\nabla$ and $\nabla^{\prime}$ are the Riemannian connections on $M=(\Gamma \backslash G, g)$ and $M^{\prime}=\left(\Gamma \backslash G,\left(\Phi^{-1}\right)^{*} g\right)$. From (2) and (3), for $\lambda \in \Lambda^{p}\left(g^{*}\right)$ and $X \in g$, we have

$$
\begin{gathered}
\Delta^{\prime}\left(\left(\Phi^{-1}\right)^{*} \lambda\right)=\left(\Phi^{-1}\right)^{*}(\Delta \lambda), \\
\nabla_{\Phi_{*} X}^{\prime}\left(\left(\Phi^{-1}\right)^{*} \lambda\right)=\left(\Phi^{-1}\right)^{*}\left(\nabla_{X} \lambda\right) .
\end{gathered}
$$

To show $\Delta^{\prime} \circ T_{p}=T_{p} \circ \Delta$ on $E^{p}(\Gamma \backslash G)$, it is enough to show $\Delta^{\prime}\left((T f)\left(\Phi^{-1}\right)^{*} \lambda\right)$ $=T_{p}(\Delta(f \lambda))$ for every $f \in C^{\infty}(\Gamma \backslash G), \lambda \in \Lambda^{p}\left(\mathrm{~g}^{*}\right)$. By (7), (13), and our previous proof for $p=0$, this reduces to showing

$$
\nabla_{\operatorname{grad}^{\prime}(T f)}^{\prime}\left(\Phi^{-1}\right)^{*} \lambda=T_{p}\left(\nabla_{\operatorname{grad} f} \lambda\right)
$$

where $\operatorname{grad}^{\prime}$ is the gradient operation relative to $g^{\prime}=\left(\Phi^{-1}\right)^{*} g$.

Let $X_{1}, \cdots, X_{n}$ be any basis of $g$ orthonormal relative to $g$. Then $\Phi_{*} X_{1}, \cdots, \Phi_{*} X_{n}$ is a basis orthonormal relative to $g^{\prime}$ and

$$
\nabla_{\mathrm{grad} f}=\sum_{j=1}^{n} X_{j} f \nabla_{X_{j}}, \quad \nabla_{\mathrm{grad}^{\prime} T f}^{\prime}=\sum_{j=1}^{n}\left(\Phi_{*} X_{j}\right)(T f) \nabla_{\Phi_{*} X_{j}}^{\prime} .
$$

Formula (15) follows directly by using these relations to expand both sides and subsequently using (12), (14), and the relation $\left(\Phi_{*} X_{j}\right)(T f)=T\left(X_{j} f\right)$ obtained by differentiation of 3.2(2). Thus we have $\Delta^{\prime}=T_{p} \circ \Delta \circ T_{p}^{-1}$ on $E^{p}(\Gamma \backslash G)$ for $1 \leqslant p \leqslant n$ and our proof is complete.

\section{Isometry classes of the isospectral manifolds}

5.1. Notation and remarks. In this section, we shall restrict the level of generality from that used above. Thus $G$ still denotes an exponential solvable group with $\Gamma$ a fixed uniform discrete subgroup of $G$ but in $\$ \S 5.2$ and 5.3 we require that all of the roots of the Lie algebra of $G$ be real while in $\$ \$ 5.4-5.6$ we insist that $G$ be nilpotent. Define

$$
D=\{\delta \in \operatorname{Aut}(G): \delta(\Gamma)=\Gamma\},
$$


and, for $g$ a left invariant Riemannian metric on $G$,

$$
K_{g}=\left\{\sigma \in \text { Aut } G: \sigma^{*} g=g\right\} \text {. }
$$

Note that $D$ is discrete and $K_{g}$ compact.

5.2. Proposition. Suppose $G$ and $\Gamma$ are as in $\$ 3.1$ and that all of the roots of the Lie algebra of $G$ are real. Let $g$ and $g^{\prime}$ be two left-invariant metrics on $G$. Then the manifolds $(\Gamma \backslash G, g)$ and $\left(\Gamma \backslash G, g^{\prime}\right)$ are isometric if and only if there exists $\Psi \in D \operatorname{Inn}(G)$ such that $\Psi^{*} g=g^{\prime}$.

Proof. By normality of $\operatorname{Inn}(G)$ in $\operatorname{Aut}(G), D \operatorname{Inn}(G)=\operatorname{Inn}(G) D$ is a subgroup of $\operatorname{Aut}(G)$. If $g^{\prime}=\Psi^{*} g$ for $\Psi=I_{x} \delta \in \operatorname{Inn}(G) D$, then $\delta$ induces a diffeomorphism $\bar{\delta}$ on $\Gamma \backslash G$. Since $I_{x}=L_{x} R_{x}^{-1}$ where $L_{x}$ and $R_{x}$ are left and right translations by $x$ and $g$ is left-invariant, $g^{\prime}=\left(R_{x}^{-1} \delta\right)^{*} g$ whence $R_{\Gamma, x}^{-1} \bar{\delta}$ : $\left(\Gamma \backslash G, g^{\prime}\right) \rightarrow(\Gamma \backslash G, g)$ is an isometry (see $\S 3.1$ for the definition of $\left.R_{\Gamma, x}\right)$. The converse is proved in [2] with the heart of the proof being the demonstration that the group of all isometries on $(G, g)$ is the semidirect product of $G$, regarded as acting by left translations on itself, and $K_{g}$.

5.3. Corollary. For $G, \Gamma$, and $g$ as in Proposition 5.2 and for $\Phi_{1}, \Phi_{2} \in$ Aut $G$, $\left(\Gamma \backslash G, \Phi_{1}^{*} g\right)$ is isometric to $\left(\Gamma \backslash G, \Phi_{2}^{*} g\right)$ if and only if there exists $\sigma \in K_{g}$ such that $\Phi_{1}^{-1} \sigma \Phi_{2} \in D \operatorname{Inn}(G)$.

Proof. By Proposition 5.2, the manifolds are isometric precisely when there exists $\Psi \in D \operatorname{Inn} G$ such that $\Phi_{2}^{*} g=\Psi^{*} \Phi_{1}^{*} g$ or, equivalently, $\sigma=\Phi_{1} \Psi \Phi_{2}^{-1} \in$ $K_{g}$.

5.4. Proposition. Let $G$ be a connected, simply-connected nilpotent Lie group with $\Gamma$ a uniform discrete subgroup of $G$ and $g$ a left-invariant metric on $G$. Let $D$ and $K=K_{g}$ be as in (1) and (2) and define

$$
\begin{aligned}
C & =\{\Phi \in \operatorname{AIA}(G): \sigma \Phi \in D \operatorname{Inn}(G) \text { for some } \sigma \in K\} \\
C_{0} & =(\operatorname{AIA}(G) \cap D) \operatorname{Inn}(G) \\
K_{0} & =K \cap D \\
K_{1} & =K \cap D \operatorname{AIA}(G) \\
S & =(K \operatorname{AIA}(G) \cap D) \operatorname{Inn}(G)=\left(K_{1} C \cap D\right) \operatorname{Inn}(G)
\end{aligned}
$$

Then

(i) $K_{1}$ is finite and $K \cap \operatorname{AIA}(G)=\{\mathrm{Id}\}$,

(ii) $S$ has a finite $\left(K_{0}, C_{0}\right)$ double coset decomposition,

(iii) $C$ is a union of finitely many right cosets of $C_{0}, C / \operatorname{Inn}(G)$ is a union of finitely many right cosets of $C_{0} / \operatorname{Inn}(G)$, and $C_{0} / \operatorname{Inn}(G)$ is a discrete subgroup of the connected, simply-connected nilpotent group $\operatorname{AIA}(G) / \operatorname{Inn}(G)$.

(iv) $S / \operatorname{Inn}(G)$ is a discrete subgroup of $\operatorname{Aut}(G) / \operatorname{Inn}(G)$. 
Proof. We shall use several times the fact that if a topological group $H$ contains a discrete subgroup $\Delta$ and a closed subgroup $L$ for which $L / L \cap \Delta$ is compact, then the image of $\Delta$ in $H / L$ is discrete. Thus we obtain the last statements in (iii) by taking $H=\operatorname{AIA}(G), L=\operatorname{Inn}(G), \Delta=\operatorname{AIA}(G) \cap D$, and noting that $L \cap \Delta$ contains the uniform discrete subgroup $\Gamma / Z$ of $\operatorname{Inn}(G) \cong$ $G / Z$.

Assume (i) for the moment. For $K_{1}=\cup_{j=1}^{r} K_{0} \sigma_{j}$ any left $K_{0}$-coset decomposition of $K_{1}$, we may then select elements $\Phi_{j} \in \operatorname{AIA}(G)$ for which $\sigma_{j} \Phi_{j} \in D$. and obtain the decompositions $S=\cup_{j=1}^{r} K_{0} \sigma_{j} \Phi_{j} C_{0}, C=\cup_{j=1}^{r} \Phi_{j} C_{0}$, thereby proving (ii), the first part of (iii), and (iv). Note that without loss of generality we may take $\sigma_{1}=\Phi_{1}=$ identity element in $\operatorname{Aut}(G)$.

The discrete set $\log \Gamma$ is cocompact in the vector space $g$ and thus spans $g$. For $X, Y \in \log \Gamma, Z=\log (\exp X \exp Y)$ and $W=\log (\exp X \exp Y \exp -$ $X \exp -Y$ ) are also in $\log \Gamma$.

From 2.1(1), we have

$$
\begin{gathered}
Z-(X+Y)=\frac{1}{2}[X, Y]+p(X, Y), \\
W-[X, Y]=q(X, Y),
\end{gathered}
$$

where $p$ and $q$ are finite sums of iterated brackets of $X$ and $Y$ each term of which contains at least three factors. For $\left\{g^{j}: j \geqslant 1\right\}$ the lower central series ideals for $g$ (see $\$ 2.1$ ), it follows from (5) that $\left[\log \Gamma, \log \Gamma \cap g^{j}\right] / \mathfrak{g}^{j+2}$ is contained in $\log \Gamma$ and by induction $\varrho_{j}=\log \Gamma \cap \mathfrak{g}^{j} / \mathfrak{g}^{j+1}$ spans $\mathfrak{g}^{j} / \mathfrak{g}^{j+1}$ for all $j$. Moreover, by (4), $\mathscr{L}_{j}$ is an additive subgroup of $\mathrm{g}^{j} / \mathrm{g}^{j+1}$. Using the remark above with $\Delta=\Gamma \cap \exp \mathfrak{g}^{j}$ and $L=\exp g^{j+1}$, it follows that $\mathcal{L}_{j}$ is discrete and consequently a lattice, i.e., uniform discrete subgroup, in the vector space $\mathfrak{g}^{j} / \mathfrak{g}^{j+1}$.

Any automorphism $\Phi$ of $G$ preserves each ideal $\mathfrak{g}^{j}$ and hence induces maps $\pi_{j}(\Phi)$ on the quotient spaces $\mathfrak{g}^{j} / \mathfrak{g}^{j+1}$. For $\Phi \in \operatorname{AIA}(G)$, Definition 2.2 implies that $\Phi_{*}-$ Id maps $\mathrm{g}^{j}$ into $\mathrm{g}^{j+1}$ and thus $\pi_{j}(\Phi)$ is the identity for all $j$. In contrast, for $\sigma \in K$, the family of maps $\pi_{j}(\sigma), 1 \leqslant j \leqslant m=$ step size of $\mathfrak{g}$, uniquely determines the orthogonal map $\sigma_{*}$ on $\mathrm{g}$. Since $\sigma_{*}$ uniquely determines $\sigma$, we have $K \cap \operatorname{AIA}(G)=\{\operatorname{Id}\}$. Now suppose $\sigma \in K, \Phi \in \operatorname{AIA}(G)$, and $\sigma \Phi \in D$. Then $\pi_{j}(\sigma \Phi)=\pi_{j}(\sigma)$ leaves the lattice $\mathcal{L}_{j}$ invariant and is orthogonal relative to the inner product on $\mathfrak{g}^{j} / \mathfrak{g}^{j+1}$ induced by $g$. Since only finitely many orthogonal linear transformations can preserve a given lattice in a Euclidean space, there are only finitely many possibilities for $\left\{\pi_{j}(\sigma): 1 \leqslant j \leqslant\right.$ $m$ \} and we conclude that $K_{1}$ is finite.

5.5. Theorem. Let $G, \Gamma$ and $g$ be as in Proposition 5.4 and suppose $\operatorname{Inn}(G)$ has codimension $d>0$ in $\operatorname{AIA}(G)$. Let $E$ be the family of isometry classes of the isospectral (by Theorem 3.4) manifolds $\left(\Gamma \backslash G, \Phi^{*} g\right)$ as $\Phi$ ranges over $\operatorname{AIA}(G)$. 
Then $E$ has a d-dimensional manifold structure arising as the double coset space of a d-dimensional Lie group by a pair of discrete subgroups, one of which is finite. Indeed, using the notation (3), $E$ may be identified with $\pi\left(K_{1}\right) \backslash \pi\left(K_{1} \operatorname{AIA}(G)\right) / \pi(S)$ for $\pi$ the canonical projection from $\operatorname{Aut}(G)$ to $\operatorname{Aut}(G) / \operatorname{Inn}(G)$.

Proof. By Corollary 5.3, if $\Phi_{1}, \Phi_{2}$ are elements of $\operatorname{AIA}(G),\left(\Gamma \backslash G, \Phi_{1}^{*} g\right)$ is isometric to $\left(\Gamma \backslash G, \Phi_{2}^{*} g\right)$ if and only if $\pi\left(\Phi_{2}\right) \in \pi\left(K_{1}\right) \pi\left(\Phi_{1}\right) \pi(S)$. Thus we have a natural identification of $E$ with $\pi\left(K_{1}\right) \backslash \pi\left(K_{1} \mathrm{AIA}(G)\right) / \pi(S)$. By Theorem 2.3 and Proposition 5.4, $\pi(S)$ is discrete and $\pi\left(K_{1} \operatorname{AIA}(G)\right)$ is the semidirect product of the finite group $\pi\left(K_{1}\right) \cong K_{1}$ and the connected, simplyconnected $d$-dimensional nilpotent Lie group $\pi(\operatorname{AIA}(G))$.

5.6. Remarks. (i) Let $\mathfrak{a}$ be any vector space complement to ad $\mathfrak{g}$ within $\mathrm{AID}(\mathrm{g})$ and $A=\exp (\mathfrak{a})$. By Theorem $2.3 \mathrm{AIA}(G)=A \operatorname{Inn}(G)$ with $A \cap$ $\operatorname{Inn}(G)=\{e\}$ and thus as topological spaces, $\mathbf{R}^{d} \approx A \approx \pi(\mathrm{AIA}(G))$. Using $A$ as a parameter set for the metrics $\Phi^{*} g, \Phi \in A$, isometry of metrics defines an equivalence relation on $A$ in which each equivalence class is discrete. Obviously $E$ is in 1-1 correspondence with these classes. It is clear that for any $\Phi \in A-C$ (for $C$ given by (3)), we can choose a continuous path $t \rightarrow \Phi_{t}$ in $A$ with $\Phi_{1}=\Phi$, such that the continuous isospectral deformation $t \rightarrow g_{t}=\Phi_{t}^{*} g$ of $g$ to $\Phi^{*} g$ has the property that $\left(\Gamma \backslash G, g_{t_{1}}\right)$ is nonisometric to $\left(\Gamma \backslash G, g_{t_{2}}\right)$ for $t_{1} \neq t_{2}$.

(ii) Calculation of $E$ is greatly simplified when, in the notation of Proposition $5.4, K_{1}$ coincides with $K_{0}$. This happens in particular when $\log \Gamma$ is a lattice in $g$ invariant under the orthogonal projection to $g^{j}$ defined by the metric $g$ for $j=1,2, \cdots, m$. In the notation of Proposition 5.4, $\log \Gamma$ is then isomorphic to the orthogonal direct sum of the lattices $\mathcal{L}_{1}, \cdots, \mathcal{L}_{m}$ and $K_{1}=K_{0}$ is the subgroup of $K$ preserving each $\mathscr{L}_{j}$.

When $K_{1}=K_{0}$, clearly $C=C_{0}$ is a subgroup of AIA $(G)$ normalized by $K_{1}$. The family of manifolds ( $\Gamma \backslash G, \Phi^{*} g$ ), $\Phi \in \operatorname{AIA}(G)$, which are nonisometric to $(\Gamma \backslash G, g)$ may be identified with the nilpotent group $\operatorname{AIA}(G) / C_{0}$ and $E$ may be identified with the orbits in this group under the action of $K_{0}$ defined by $\Phi C_{0} \rightarrow\left(\sigma \Phi \sigma^{-1}\right) C_{0}, \sigma \in K_{0}$.

In the following examples, we shall frequently insist that $\Gamma$ and $g$ satisfy the above simplifying condition.

5.7. Examples. (i) Let $G$ be the group of Example 2.4(i) and define $\Gamma=\exp \mathcal{L}$ for $\mathcal{E}$ the lattice in $\mathrm{g} \approx \mathbf{R}^{6}$ with lattice basis $\left\{X_{i}, Y_{i}, \frac{1}{2} Z_{i}: i=1,2\right\}$. By 2.1(1) and the defining bracket relations for $g, \Gamma$ is a uniform discrete subgroup of $G$. The almost inner derivations $\varphi_{1}$ and $\varphi_{2}$ map $\Gamma$ into $\Gamma$ and $\operatorname{AIA}(G) \cap D$ is the exponential of the lattice in $\mathbf{R}^{6} \approx \operatorname{AID}(g)$ spanned by $\varphi_{i}$, ad $X_{i}$, ad $Y_{i}, i=1,2$. Thus the group $\operatorname{AIA}(G) / C_{0}$ of 5.6(ii) becomes simply the 2-torus $\operatorname{span}_{\mathbf{R}}\left\{\varphi_{1}, \varphi_{2}\right\}$ 
modulo $\operatorname{span}_{\mathbf{z}}\left\{\varphi_{1}, \varphi_{2}\right\}$. If we take $g$ so that our basis above for $\mathcal{L}$ is orthonormal, a routine computation shows that the $K_{0}$-orbit of the point $\left(t_{1}, t_{2}\right)=t_{1} \varphi_{1}$ $+t_{2} \varphi_{2}+\mathbf{Z} \varphi_{1}+\mathbf{Z} \varphi_{2}$ consists of the points $\left(\epsilon_{1} t_{1}, \epsilon_{2} t_{2}\right)$ for $\epsilon_{1}, \epsilon_{2} \in\{ \pm 1\}$.

(ii) Let $G$ be the group of Example 2.4(ii) with $\log \Gamma=\operatorname{span}_{\mathrm{z}}\left\{X_{1}, X_{2}, Y_{i}, \frac{1}{2} Z_{i}\right.$ : $i=1,2,3\}$. We know that all almost inner derivations of $g$ rational with respect to the chosen basis are inner. Since $\Phi \in \operatorname{AIA}(G) \cap D$ implies that $\varphi=\Phi-$ Id is a rational almost inner derivation, the group $C_{0}$ of Proposition 5.4 is simply $\operatorname{Inn}(G)$ and the group $\operatorname{AIA}(G) / C_{0}$ of $5.6(\mathrm{ii})$ is therefore isomorphic to $\mathbf{R}^{2}$. Depending on the choice of $g, E$ either remains $\mathbf{R}^{2}$ or consists of orbits in $\mathbf{R}^{2}$ under the action of a finite group. In this example as well as the one above, similar conclusions hold if $\log \Gamma$ is redefined by a basis whose elements are arbitrary rational combinations of the original basis.

(iii) Let $G$ be the exponential solvable group whose Lie algebra $g$ was constructed in Example 2.4(iv). We first construct a uniform discrete subgroup of $G$. Choose any matrix $\left[\begin{array}{c}\alpha \beta \\ \gamma \delta\end{array}\right] \in \operatorname{SL}(2, \mathbf{Z})$ with $\alpha+\delta>2$ and let

$$
\lambda_{ \pm}=\left(\frac{\alpha+\delta}{2}\right) \pm \sqrt{\left(\frac{\alpha+\delta}{2}\right)^{2}-1}
$$

Then $\left[\begin{array}{cc}\lambda_{+} & 0 \\ 0 & \lambda_{-}\end{array}\right]$is similar to $\left[\begin{array}{c}\alpha \\ \gamma\end{array}\right]$. It follows that there exist elements $U_{3}=$ $a X_{3}+b Y_{3}, V_{3}=c X_{3}+d Y_{3}$ with $a d-b c=1$ such that $e^{t_{0} \text { ad } H}$ leaves $\mathcal{L}_{3}=$ $\operatorname{span}_{\mathbf{z}}\left\{U_{3}, V_{3}\right\}$ invariant for $t_{0}=\log \lambda_{+}$. Now define $\Gamma$ as the subgroup of $G$ generated by $\left\{\exp \left(n t_{0} H\right): n \in \mathbf{Z}\right\}$, $\exp \mathcal{L}_{3}$, and $\exp \mathcal{L}$ for $\mathcal{L}$ as in 5.7(i). Since $\left[U_{3}, V_{3}\right]=Z_{1} \in \mathcal{L}$, it is easy to check that $\Gamma$ is a uniform discrete subgroup of $G$. Let $A=\left\{\alpha\left(t_{1}, t_{2}\right)=\exp \left(t_{1} \psi_{1}+t_{2} \psi_{2}\right):\left(t_{1}, t_{2}\right) \in \mathbf{R}^{2}\right\}$. By an easy direct calculation (note Theorem 2.3 does not apply) $\operatorname{AIA}(G)=A \cdot \operatorname{Inn}(G)$. Let $g$ be the left invariant metric on $G$ for which the basis $\left\{H, X_{1}, X_{2}, X_{3}, Y_{1}, Y_{2}, Y_{3}, Z_{1}, Z_{2}\right\}$ is orthonormal. Although $G$ is not nilpotent, the group $K_{1}$ is still finite. To see this, note first that for $\sigma \in K_{g}$, $\sigma_{*}(H)= \pm H$ since $\mathbf{R} H$ is the orthogonal complement of the $\sigma_{*}$-invariant subspace nilrad(g). If $\sigma_{*} H=H$, then $X_{3}$ and $Y_{3}$ must be eigenvectors of $\sigma_{*}$ (since $\sigma_{*} X_{3}=\sigma_{*}\left[H, X_{3}\right]=\left[H, \sigma_{*} X_{3}\right]$, etc.) while if $\sigma_{*} H=-H$, then $\sigma_{*}$ permutes $\mathbf{R} X_{3}$ and $\mathbf{R} Y_{3}$. Thus $\sigma_{*}$ leaves invariant $\mathfrak{m}=\operatorname{span}_{\mathbf{R}}\left\{H, X_{3}, Y_{3}\right\}$ and there are only finitely many possibilities for $\left.\sigma_{*}\right|_{\mathfrak{m}}$. If $\sigma$ is in $K_{1}$ with $\sigma \Phi \in D$ and $\Phi \in \operatorname{AIA}(G)$, then $\sigma_{*}$ and $\Phi_{*}$ leave invariant

$$
\mathrm{m}^{\perp}=\operatorname{span}_{\mathbf{R}}\left\{X_{1}, X_{2}, Y_{1}, Y_{2}, Z_{1}, Z_{2}\right\} .
$$

However, on $\mathrm{m}^{\perp}, \Phi_{*}-$ Id is nilpotent and maps $\mathrm{m}^{\perp}$ to $z=\operatorname{span}_{\mathbf{R}}\left\{Z_{1}, Z_{2}\right\}=$ center of $g$ and the arguments given in the proof of Proposition 5.4 apply to yield only finitely many possibilities for $\left.\sigma_{*}\right|_{\mathfrak{m}^{\perp}}$. 
Clearly $\alpha\left(t_{1}, t_{2}\right) \in D$ if and only if $t_{1}, t_{2} \in \mathbf{Z}$. By the obvious analogs of our arguments above in 5.4-5.7(i), it follows that the isometry classes of the isospectral manifolds $\left(\Gamma \backslash G, \Phi^{*} g\right), \Phi \in A$, are parametrized by equivalence classes in the 2-torus under an equivalence relation in which each equivalence class has finitely many elements.

\section{References}

[1] M. Berger, P. Gauduchon \& E. Mazet, Let spectre d'une variété Riemannienne, Lecture Notes in Math., Vol. 194, Springer, Berlin and New York, 1971.

[2] C. S. Gordon \& E. N. Wilson, Isometry groups of solvmanifolds, submitted for publication.

[3] V. Guillemin \& D. Kazhdan, Some inverse spectral results for negatively curved two manifolds, Topology 19 (1980) 301-312.

[4] S. Helgason, Differential geometry, Lie groups, and symmetric spaces, Academic Press, New York, 1978.

[5] A. Ikeda, On the spectrum of a Riemannian manifold of positive constant curvature, Osaka J. Math. 17 (1980) 75-93; 691-702.

[6] C. C. Moore, Representations of solvable and nilpotent groups and harmonic analysis on nil and solvmanifolds, Proc. Sympos. Pure Math., Vol. 26, Amer. Math. Soc., Providence, RI, 1973, 3-44.

[7] L. Pukanszky, Leçons sur les représentations des groupes, Dunod, Paris, 1966.

[8] M. S. Raghunathan, Discrete subgroups of Lie groups, Springer, Berlin and New York, 1972.

[9] G. de Rham, Variétés différentiables, Hermann, Paris, 1960.

[10] U. Simon \& H. Wissner, Geometry of the Laplace operator, preprint, Technische Universität Berlin.

[11] H. Urakawa, Bounded domains which are isospectral but not isometric, Ann. Sci. Ėcole Norm. Sup. (4) 15 (1982) 441-456.

[12] M.-F. Vignéras, Variétés riemanniennes isospectrales et non isométriques, Ann. of Math. 112 (1980) 21-32. 